CIplus

Band 1/2018

\title{
Optimization via Multimodel Simulation
}

A New Approach to Optimization of Cyclone Separator Geometries

Thomas Bartz-Beielstein, Martin Zaefferer, Quoc Cuong Pham 
This is a pre-print of an article published in Structural and Multidisciplinary Optimization .

The final authenticated version is available online at:

https://doi.org/10.1007/s00158-018-1934-2 


\title{
Optimization via Multimodel Simulation
}

\section{A New Approach to Optimization of Cyclone Separator Geometries}

\author{
Thomas Bartz-Beielstein • Martin Zaefferer • Quoc Cuong Pham
}

Received: date / Accepted: date

\begin{abstract}
Increasing computational power and the availability of 3D printers provide new tools for the combination of modeling and experimentation. Several simulation tools can be run independently and in parallel, e.g., long running computational fluid dynamics simulations can be accompanied by experiments with 3D printers. Furthermore, results from analytical and data-driven models can be incorporated. However, there are fundamental differences between these modeling approaches: some models, e.g., analytical models, use domain knowledge, whereas data-driven models do not require any information about the underlying processes. At the same time, data-driven models require input and output data, but analytical models do not. Combining results from models with different input-output structures might improve and accelerate the optimization process. The optimization via multimodel simulation (OMMS) approach, which is able to combine results from these different models, is introduced in this paper.

Using cyclonic dust separators as a real-world simulation problem, the feasibility of this approach is demonstrated and a proof-of-concept is presented. Cyclones are popular devices used to filter dust from the emit-
\end{abstract}

Thomas Bartz-Beielstein (corresponding author)

Technische Hochschule Köln

Steinmüllerallee 1, 51643 Gummersbach, Germany

Tel.: +49-2261-8196-6391

Fax: +49-2261-8196-6666

E-mail: thomas.bartz-beielstein@th-koeln.de

ORCID: 0000-0002-5938-5158

Martin Zaefferer

Technische Hochschule Köln

Steinmüllerallee 1, 51643 Gummersbach, Germany

ORCID: 0000-0003-2372-2092

Quoc Cuong Pham

Technische Hochschule Köln

Steinmüllerallee 1, 51643 Gummersbach, Germany ted flue gases. They are applied as pre-filters in many industrial processes including energy production and grain processing facilities. Pros and cons of this multimodel optimization approach are discussed and experiences from experiments are presented.

Keywords Combined simulation · multimodeling · simulation-based optimization $\cdot$ metamodel $\cdot$ multifidelity optimization · stacking · response surface methodology · 3D printing · computational fluid dynamics

\section{Introduction}

Modeling allows the estimation of system performance under new conditions as well as the comparison of different operating conditions and parameterizations, e.g., new geometries. This article describes different model types, namely analytical, surrogate, computational fluid dynamics (CFD), and 3D printing models. Because every modeling approach has its pros and cons, a combination, which uses information from several models at the same time, might be beneficial. Starting with mathematical modeling, we will describe different modeling approaches first.

Loosely speaking, mathematical modeling is "the link between mathematics and the rest of the world" (Meerschaert 2013). Mathematical modeling can be performed using analytical and numerical models: Analytical models are mathematical models that have a closed form solution, i.e., the solution to the equations used to describe changes in a system can be expressed as a mathematical analytic function. Nelson (1995) refers to analytical models as "rough-cut models", i.e., mathematically solvable and typically less detailed models. 
Numerical (simulation) models are mathematical models that use some sort of numerical time-stepping methods such as Newton's method to simulate the model's behavior over time. In contrast to analytical models, solutions of simulation models are usually presented as tables or plots. Simulation is a widely used method for studying complex real-world systems, because many systems cannot be completely described by analytical models and experimentation with the real system is infeasible or expensive (Law 2007).

Nowadays, CFD simulation is a well established technique. It is also used in many studies, which describe the topic discussed in this publication: the optimization of cyclone separator geometries (Hoffmann and Stein 2007; Elsayed and Lacor 2010).

Over the last decades, surrogate models, also known as metamodels, gained importance (Jin et al. 2001; BartzBeielstein and Zaefferer 2017). They are build from and then used instead of the underlying real processes or simulation models. Popular metamodelling techniques include regression, radial basis functions, and Kriging (Santner et al. 2003; Kleijnen 2008).

$3 D$-printing is a popular modeling technique. It is commonly used to validate the results, e.g., a certain geometry, from CFD simulations. Recently, 3D-printing was integrated into the optimization via simulation loop (Preen and Bull 2014).

Although the model based approach can be considered a success story, it also causes some problems. Several critical issues in simulation studies are related to errors (Nelson 1995). These errors can be due to bias (e.g., initial-condition effects) or to problems with the pseudorandom-number generators. If feasible, an analytical analysis is in many cases preferable to simulation, because of the lack of sampling error. Simulation models can also be computationally demanding, because each simulation describes only one single setting. Therefore several repeats with varying input data are necessary, whereas an analytical model allows the calculation of the exact characteristics of the system for several settings.

Furthermore, an inappropriate level of model detail, failure to collect adequate system data, and using wrong performance indicators for comparisons are common pitfalls in both analytical and numerical simulation studies (Law 2007).

Many textbooks describe methods for finding the best model, but do not discuss the combination of several models. Nelson (1995) stated that textbooks "tend to give the impression that there is a unique best model of any real or conceptual system. This is not correct." More than one type of model will be used in practice. The increasing computational power and the avail- ability of 3D printers provide tools for new modeling approaches. Several simulations can be run in parallel, e.g., long running CFD simulations can be accompanied by experiments with $3 \mathrm{D}$ printers, whereas the analytical model is evaluated as a baseline. Combinations of the following approaches are possible: (i) analytical models, (ii) numerical simulation, (iii) surrogate models, (iv) lab experiments, and (v) field experiments. The central question in this context is: Are there any benefits in combining different simulation approaches and can the weakness of one approach be compensated by other approaches? To answer this question, an approach for combining these heterogenous results is necessary. This article presents a new approach for handling several simulation models in parallel, which will be referred to as optimization via multimodel simulation (OMMS). The OMMS approach can be used as the central part of the well-established optimization via simulation methodology ( $\mathrm{Fu} 1994)$. To exemplify OMMS, a real-world application is used: cyclone dust collectors. This article presents results from an experimental study, which can be regarded as a proofof-concept for OMMS. For the experiments, we have chosen a combination of four different modeling approaches:

(M-A) analytical,

(M-C) CFD simulation,

(M-S) surrogate (metamodels), and

(M-P) 3D printing models.

This paper is structured as follows: Section 2 describes related work. Cyclone dust absorbers are briefly described in Section 3. Section 4 presents the OMMS loop. Section 5 compares results from different modeling approaches. Experimental results based on these modeling approaches are presented in Section 6. How to combine results from various models via ensemble building is shown in Section 7. Finally, Section 8 gives a conclusion and an outlook.

\section{Related Work}

The idea of using different models with different resolutions has been discussed in the literature for many years. Zeigler and Oren (1986) describe multiple levels of model aggregation (resolution, abstraction). These levels depend on the objectives, knowledge, and the available budget (resources, e.g., time). Fishwick and Zeigler (1992) present a formalism and a methodology for developing multiple, cooperative models of physical systems from qualitative physics. Barzier and Perry (1991) describe a two-level modeling approach for developing simulation models in the shipbuilding industry. Chaudhuri et al. (2015) describe a flapping wing 
optimization task. They use multiple surrogates, multiple infill criteria, and multiple points for the same experimental data set. Kazemi et al. (2016) use different machine learning approaches to create simple and reliable models for predicting granule size distributions. An iterative procedure assisted by cross validation was implemented to find out the best model among thousands. The cyclone modeling, simulation, and optimization approach presented in our study is related to the work from Preen and Bull (2014), who optimized verticalaxis wind turbines using miniaturized 3D-printed wind turbines.

Yang (2003) states that selection of one model can be better when the errors in prediction are small and that the model combination works better when the errors are large. Simpson et al. (2012) present a thoughtful review of several multimodel approaches. They state that "the use of multiple surrogates (i.e., a set of surrogates and possibly a weighted average surrogate) is very appealing in design optimization due to the fact that the best surrogate may not lead to the best result; and complementary because fitting many surrogates and repeating optimizations is cheap compared to cost of simulation." They also describe a multidisciplinary approach which is not directly comparable to OMMS, because independent models for different subsystems are combined rather than integrating several models of the same system.

Furthermore, co-Kriging, which is a popular method that combines results from fine and coarse grained models, can be mentioned in this context (Forrester et al. 2007). Typically, co-Kriging tries to combine data from models which have different fidelity, e.g., a fine model that is expensive to compute and a less accurate, coarse model, which is cheaper to compute. In contrast to single-fidelity Kriging models, co-Kriging attempts to learn the correlation between the coarse and fine model, thus being able to exploit the larger amount of data derived from the coarse model to improve the representation of the expensive, fine model. This could be used for the meta-modeling step, especially when different levels of fidelity are available.

In general, there are two options to deal with multiple models: (i) selection of the best model and (ii) combination of results from several models. Most approaches try to select one model, whereas OMMS combines results from several models using stacked regression (Wolpert 1992; Bartz-Beielstein 2016). Our study presents an integrated simulation and experimentation methodology on various scales (or layers).

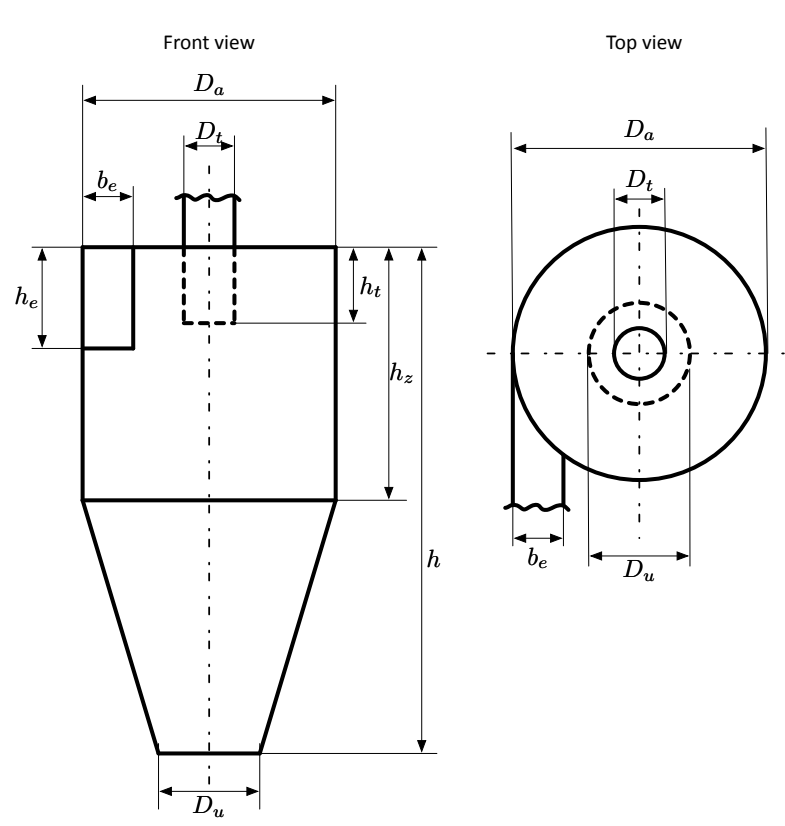

Fig. 1 Standard geometry of the cyclone considered in this study. The corresponding geometry parameters, $\mathbf{x}_{g}$, are described in Table 1.

\section{Cyclone Dust Collectors}

Cyclones are used in oil and gas, iron and steel, chemical and food industry to filter a maximal amount of dust from flue gas (Hoffmann and Stein 2007). They can be applied in extremely harsh and demanding environments, but show a relatively low separation compared to electrostatic dust collectors. An efficient cyclone requires the optimization of its geometry parameters, which are shown in Figure 1. Even with today's modern tools, the complexity of cyclone behavior is such that experimental studies are necessary for a solid understanding of the phenomena governing their behavior. The cyclone geometry can be specified by the parameter vector, $\mathbf{x}_{g}$, with the following entries: inlet width $b_{e}$, body diameter $D_{a}$, diameter of the vortex finder $D_{t}$, diameter of the dust exit $D_{u}$, total height $h$, inlet height $h_{e}$, vortex finder immersion $h_{t}$, and cylinder height $h_{z}$. In addition to these geometry parameters, $\mathbf{x}_{g}$, the specification of the operating parameters, $\mathbf{x}_{p}$, is necessary. The geometry and process parameter sets are shown in Table 1. We will concentrate in this study on the collection efficiency as specified in Löffler (1988), which will be explained in Section 5.1. 
Table 1 Nomenclature from Löffler (1988). Values (L, M, S) refers to the values of the geometry parameters $\mathbf{x}_{g}$ for the Löffler, Muschelknautz E., and Stairmand high efficiency cyclones, respectively. The vortex finder immersion, $h_{t}$, is modified for every cyclone geometry. The type " $\mathbf{x}_{p}$ " denotes operating parameters. Parameter values, which depend on other values, are labeled as "*" in the Type column. Parameters to be optimized are labeled in the last column.

\begin{tabular}{|c|c|c|c|c|c|}
\hline Parameter & Units & Values $(\mathrm{L}, \mathrm{M}, \mathrm{S})$ & Type & Description & Optimized \\
\hline$b_{e}$ & $\mathrm{~mm}$ & $12.8 ; 9.92 ; 7.97$ & $\mathbf{x}_{g}$ & inlet width & yes \\
\hline$D_{a}$ & $\mathrm{~mm}$ & $80.64 ; 116.48 ; 39.97$ & $\mathbf{x}_{g}$ & body diameter & yes \\
\hline$D_{t}$ & $\mathrm{~mm}$ & $26.88 ; 29.12 ; 19.98$ & $\mathbf{x}_{g}$ & diameter of the vortex finder & yes \\
\hline$D_{u}$ & $\mathrm{~mm}$ & $26.88 ; 39.04 ; 15.04$ & $\mathbf{x}_{g}$ & diameter of the dust exit & yes \\
\hline$h$ & $\mathrm{~mm}$ & $160 ; 160 ; 160$ & $\mathbf{x}_{g}$ & total height of the cyclone & yes \\
\hline$h_{e}$ & $\mathrm{~mm}$ & $38.4 ; 29.6 ; 19.98$ & $\mathbf{x}_{g}$ & inlet height & yes \\
\hline$h_{t}$ & $\mathrm{~mm}$ & $0 ; 35 ; 44$ & $\mathbf{x}_{g}$ & vortex finder (outlet pipe) immersion & yes \\
\hline$h_{z}$ & $\mathrm{~mm}$ & $44.8 ; 29.64 ; 59.95$ & $\mathbf{x}_{g}$ & cylinder height & yes \\
\hline$r_{a}$ & $\mathrm{~mm}$ & $D_{a} / 2$ & $*$ & cyclone radius & no \\
\hline$r_{i}$ & $\mathrm{~mm}$ & $D_{t} / 2$ & $*$ & radius of the vortex finder & no \\
\hline$h_{i}$ & $\mathrm{~mm}$ & $h-h_{t}$ & $*$ & height of the imaginary cylinder CS & no \\
\hline$r_{e}$ & $\mathrm{~mm}$ & $r_{a}-b_{e} / 2$ & $*$ & mean inlet pipe radius & no \\
\hline$F$ & - & $F_{e} / F_{i}$ & $*$ & ratio between inlet and outlet area & no \\
\hline$F_{e}$ & $\mathrm{~mm}^{2}$ & $h_{e} \times b_{e}$ & * & inlet area & no \\
\hline$F_{i}$ & $\mathrm{~mm}^{2}$ & $\pi \times r_{i}^{2}$ & $*$ & outlet area & no \\
\hline$v_{e}$ & $\mathrm{~ms}^{-1}$ & 20 & $\mathbf{x}_{p}$ & inlet velocity & no \\
\hline$\lambda_{g}$ & - & 0.005 & $\mathbf{x}_{p}$ & load-free friction coefficient & no \\
\hline$\mu$ & $\mathrm{Pa} \mathrm{s}$ & $1.8 \times 10^{-5}$ & $\mathbf{x}_{p}$ & viscosity & no \\
\hline$\varrho_{f}$ & $\mathrm{~kg} / \mathrm{m}^{3}$ & 1.2000 & $\mathbf{x}_{p}$ & gas density & no \\
\hline$\varrho_{p}$ & $\mathrm{~kg} / \mathrm{m}^{3}$ & 2700 & $\mathbf{x}_{p}$ & particle density & no \\
\hline$c_{\text {roh }}$ & $\mathrm{kg} / \mathrm{m}^{3}$ & 0.061 & $\mathbf{x}_{p}$ & raw gas concentration & no \\
\hline$B$ & - & $B=c_{\text {roh }} / \rho_{f}$ & $*$ & mass load & no \\
\hline$v_{i}$ & $\mathrm{~ms}^{-1}$ & $\dot{V} /\left(\pi r_{i}^{2}\right)$ & * & velocity vortex finder (outlet pipe) & no \\
\hline$v_{r}\left(r_{i}\right)$ & $\mathrm{ms}^{-1}$ & Eq. (1) & $*$ & radial gas velocity on the outlet pipe & no \\
\hline$v_{\varphi i}$ & $\mathrm{~ms}^{-1}$ & Eq. (2) & $*$ & tangential velocity at CS & no \\
\hline$\dot{V}$ & $\mathrm{~m}^{3} / \mathrm{h}$ & $F_{e} \times v_{e}$ & * & volumetric flowrate through the cyclone & no \\
\hline$\lambda$ & - & $\lambda_{g}(1+2 \sqrt{B})$ & $*$ & wall friction factor; friction coefficient & no \\
\hline
\end{tabular}

\section{Optimization via Multimodel Simulation in the Loop}

In the optimization via simulation setting, the goal is to perform runs of the simulation model in an efficient manner and to determine those input variables, which result in an optimal (or near optimal) solution (Fu 1994). The OMMS approach extends the standard optimization via simulation setting by integrating results from several model types. In contrast to mathematical models, which usually require some input values only, datadriven models require the specification of input and output values. To clarify the data flow and model building process in the OMMS approach, the following model categories will be used:

- $X$-models use input parameters, e.g., geometry and process parameters.

- XY-models use the input parameters as well as the corresponding output values, e.g., collection efficiency. So, the analytical (M-A), CFD (M-C), and 3D printing (M-P) models are considered as $X$-models, whereas the surrogate (M-S) models are $X Y$-models.

The general concept of OMMS is illustrated in Figure 2. Here, we consider the optimization of the cyclone's geometry parameters, which should be distin- guished from the process parameters. It consists of the following steps:

(S-1) Select an initial design. Set $t=1$, where $t$ denotes the number of parameter sets. The first set of geometry parameters, $\mathbf{x}_{g}^{(t)}$, is generated.

(S-2) Specify the process parameters $\mathbf{x}_{p}$. They are not changed during the optimization.

(S-3) Select $X$-models (e.g., CFD, analytical). In addition to the geometry and process parameter sets, further parameters might be necessary for each separate model. These model specific parameters will be referred to as $\mathbf{x}_{m}$. For example, the CFD simulator requires the specification of parameters for heat transfer, surface properties, damping, collision, and radiation. These parameters are not used in other simulation models. They are not changed during the optimization. The set $\mathbf{x}^{(t)}=\left(\mathbf{x}_{g}^{(t)}, \mathbf{x}_{\mathbf{p}}, \mathbf{x}_{\mathbf{m}}\right)$ will be used to build the $X$-models.

(S-4) Build $X$-models. For building these models no information about the dependent (output) variables $y$ is needed. In this step, one or several models $\left(f_{1}\right.$, $\left.\ldots, f_{p}\right)$ from the set of $X$-models, which comprehends 3D-printed objects, analytical model formulas, or CFD simulation models, are generated. The 


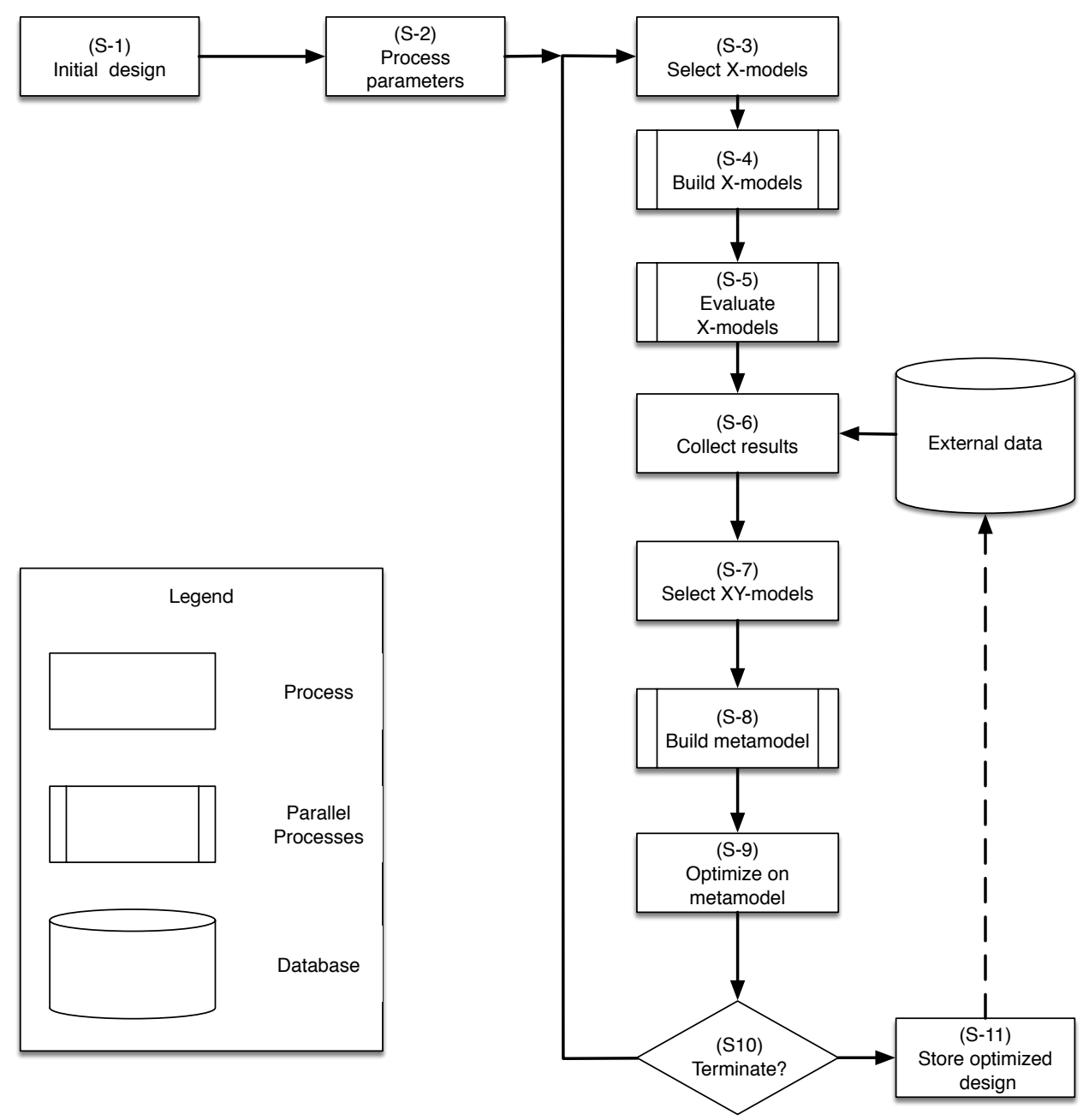

Fig. 2 Optimization via multimodel simulation in the loop. Several simulation models are used in parallel. Elements of the first set of models, i.e., during steps (S-3), (S-4), and (S-5), can be one or several CFD simulators, analytical models, or experiments based on 3D-printed objects. Results from these different models are collected and optionally combined with additional results, which were stored in a database. The second set of models is build during step (S-8). Models from the second set are classical surrogate models, e.g., neural networks, linear regression models, or Kriging models. Because simulation results, i.e., $y$-values are available at this stage of the multimodel simulation process, a broader set of models can be used than during the first steps (S-3) to (S-5). Results from these models can combined in several ways. We describe an approach that is based on stacked generalization (Wolpert 1992). Optimization is performed on the stacked model (S-9).

construction process results in several models, which use the same set of parameters $\mathbf{x}^{(t)}$.

(S-5) Evaluate models. The models are evaluated, i.e., each model generates an output: $f_{j}: \mathbf{x}^{(t)} \rightarrow y_{j}^{(t)}$. Note, some models generate a deterministic output, e.g., CFD models, whereas other, e.g., 3D-printed models, generate stochastic (noisy) outputs. Therefore, repeats should be considered for the stochastic models, to improve the quality of the measured values (Law 2007; Haftka et al. 2016).

(S-6) Collect results. Besides the set of pairs $\left\{\left(\mathbf{x}^{(k)}, y_{j}^{(k)}\right)\right\}$, for $k=1, \ldots, t$ and $j=1, \ldots, p$, additional results $\left\{\left(\mathbf{x}^{(m)}, y_{l}^{(m)}\right)\right\}$, for $m=1, \ldots, s$ and $l=1, \ldots, q$, e.g., from historical data or data from the literature, can be used in the construction of the metamodels.

(S-7) Select $X Y$-models. $X Y$-models use the parameter set, $\mathbf{x}^{(k)}=\left(\mathbf{x}_{g}^{(k)}, \mathbf{x}_{\mathbf{p}}\right)$ as well as the corresponding output values $y_{i}^{(k)}$ for model building, with $k=$ $1, \ldots,(t+s)$. In general the number of design points $(p+s)$ is required to be large enough to allow building reasonable models.

(S-8) Build metamodel. An ensemble engine builds a metamodel by combining information from several models, say $F_{i}$. It implements stacking methods. The metamodel will be referred to as $F^{*}$. The ensemble engine selects several models from the huge variety of surrogate models (e.g., random forest, or 
Kriging). These serve as basic or level-0 models. Cross validation is used to build an ensemble model from the portfolio of level-0 models. The level-1 training algorithm is typically a relatively simple linear model. Instead of stacking, a weighted combination of models $F_{i}$ or co-Kriging can be used. If models of similar fidelity are combined, we would suggest to employ to stacking. In a mixed case, a coKriging model could be integrated into a stacked metamodel (i.e., as a single model $F_{i}$ ). Other ensemble techniques may also be applicable, e.g., bagging or boosting (Murphy 2012). However, stacking is very effective even when combining only few, strong learners and it provides additional information, e.g., the contribution of each of the combined models to the ensemble.

(S-9) Optimize on the metamodel. The model $F^{*}$ is used as a surrogate for performing the optimization step. The optimization results in a new set of promising geometry parameters, which will be evaluated in the following step. The counter for the number of parameter sets $t$ is incremented and the new design can be referred to as $\mathbf{x}_{g}^{(t)}$. Instead of increasing $t$ by one, several new design points, e.g., from models with different run times, can be added to the parameter set.

(S-10) Check the termination criterion.

(S-11) Store the optimized design. Optionally, it can be added to a database.

\section{Modeling Approaches}

To exemplify the OMMS approach, four different modeling approaches are described in the following: analytical (M-A), surrogate (M-S), CFD simulation (M-C) and $3 \mathrm{D}$ printing $(\mathrm{M}-\mathrm{P})$.

\subsection{The Analytical Model (M-A)}

A broad variety of analytical models intended to predict cyclone separation performance exists in the literature (Löffler 1988; Overcamp and Mantha 1998; Hoffmann and Stein 2007). The analytical approach developed by Barth (1956) and Muschelknautz (1972) can be considered as standard. It will be referred to as the Bart-Muschelknautz method of modeling. This method is based on the assumption that a particle carried by the vortex is influenced by two forces: a centrifugal force and a flow resistance. They are expressed at the outlet pipe radius $r_{i}$ where the highest tangential velocity occurs. Some assumptions can be considered reasonable enough to obtain a good compromise between accurate prediction and simplification of the equations, e.g., the particles are spherical, the particle motion is not influenced by the presence of neighboring particles, and the radial force on the particle is given by Stokes's law.

Based on the geometry and operating parameters from Table 1, the following calculations can be performed. The equilibrium-orbit model assumes a cylindrical control-surface (CS), which is constructed by extending the vortex finder wall to the bottom of the cyclone. Let $h_{i}$ denote the height of the CS. The radial velocity at $r_{i}$ equals to:

$v_{r}\left(r_{i}\right)=\frac{\dot{V}}{2 \pi r_{i}\left(h-h_{t}\right)}$.

For a given mass load $B=c_{\text {roh }} / \rho_{f}$, the wall friction factor $\lambda$ can be calculated as $\lambda=\lambda_{g}(1+2 \sqrt{B})$. The correction factor $\alpha$ for contraction is equal to:

$\alpha=1.0-\left(0.54-\frac{0.153}{F}\right) B_{e}^{\frac{1}{3}}$.

Using the outlet pipe velocity $v_{i}=\frac{\dot{V}}{\pi r_{i}^{2}}$, Barth (1956) derived the following equation

$\frac{v_{\varphi i}}{v_{i}}=U=\frac{1}{F \alpha \cdot \frac{r_{i}}{r_{e}}+\lambda \cdot \frac{h}{r_{i}}}=\frac{r_{i} r_{e} \pi}{\alpha F_{e}+h_{i} r_{e} \pi \lambda}$.

These velocities are used to determine the collection efficiency. The equilibrium-orbit model is based on a force balance on a particle that is rotating at radius $r_{i}$. Small particles leave the cyclone through the vortex finder, whereas large particles are moving to the cyclone wall. The cut size, $x_{50}$, plays a central role in these calculations. For cyclones, particles of size $x_{50}$ have a $50-$ 50 chance of being captured, smaller particles are less likely to be captured, larger particles are more likely to be captured. The forces acting on a particle rotating on the CS, which is assumed to separate the outer region of downward flow from the inner region of upward flow, are (i) the centrifugal force acting outward with a magnitude of $\pi x^{3} \rho_{p} v_{\varphi i}^{2} /\left(6 r_{i}\right)$ and (ii) the Stokesian drag acting inward $3 \pi x \mu v_{r}\left(r_{i}\right)$. By equating these forces, Barth (1956) developed an analytical model for the cut size as follows:

$x_{50}=\sqrt{\frac{18 \mu v_{r}\left(r_{i}\right) r_{i}}{\left(\varrho_{p}-\varrho_{f}\right) v_{\varphi i}^{2}}}$.

The fractional efficiency curve assigns an efficiency to the particle diameter. It is described by

$T(x)=\left(1+\frac{2}{\left(\frac{x}{x_{50}}\right)^{3.564}}\right)^{-1.235}$, 
Table 2 Particle size distribution table. Values correspond to the dust used in the 3D printing experiments.

\begin{tabular}{llllr}
\hline Particle Size $x[\mu m]$ & $\Delta x$ & $\begin{array}{l}\text { Mean } \\
\tilde{x} \\
\end{array}$ & $\Delta Q_{e}(x)$ & Cumulative \\
& & & \\
\hline \hline $0-1$ & 1 & 0.5 & 0.1 & 0.1 \\
$1-2.7$ & 1.7 & 1.85 & 0.1 & 0.2 \\
$2.7-5.5$ & 2.8 & 4.1 & 0.1 & 0.3 \\
$5.5-8.7$ & 3.2 & 7.1 & 0.1 & 0.4 \\
$8.7-12.7$ & 4 & 10.7 & 0.1 & 0.5 \\
\hline $12.7-16.9$ & 4.2 & 14.8 & 0.1 & 0.6 \\
$16.9-21.2$ & 4.2 & 19 & 0.1 & 0.7 \\
$21.2-25.4$ & 4.2 & 23.25 & 0.1 & 0.8 \\
$25.4-30.8$ & 5.4 & 28.1 & 0.1 & 0.9 \\
$30.8-63$ & 31.2 & 46.9 & 0.1 & 1.0 \\
\hline \hline
\end{tabular}

where $x$ is the particle size. The overall collection efficiency $E$ is predicted according to:

$E=\int_{x_{\min }}^{x_{\max }} T(x) q_{e}(x) d x \approx \sum_{x_{\min }}^{x_{\max }} T\left(\tilde{x}_{i}\right) \Delta Q_{e}\left(x_{i}\right)$,

where $x_{\min }$ is the lower bound of the particle size, $x_{\max }$ is the upper bound of the particle size, $\tilde{x}_{i}$ is the mean particle size in each fraction, $\Delta Q_{e}\left(x_{i}\right)$ is the change in distribution of particle sizes and $q_{e}(x)=\Delta Q_{e}\left(x_{i}\right) / \Delta x_{i}$. The particle size distribution table, which was used in our studies, is shown in Table 2. Results from our collection efficiency $E$ calculations for models from the literature and for models used in our experiments are shown in Table 3 and Table 5, respectively. The corresponding function is available in the $\mathrm{R}$ package SPOT as funCyclone().

\subsection{CFD Simulations (M-C)}

Computational Fluid Dynamics simulations have proven to be useful for studying the fluid and particle flows in cyclones (Hoekstra et al. 1999). They have clear advantages for understanding the details of the flow in cyclones, but also limitations in terms of modeling cyclone separation performance accurately (Hoffmann and Stein 2007). Numerical simulations are performed by solving the unsteady-state, three-dimensional Reynolds averaged Navier-Stokes (RANS) equations combined with a closure model for the turbulent stresses and the large eddy simulation approach.

The CFD simulations were carried out with the open source software OpenFOAM, which has been developed for solving numerical problems (Konan and Huckaby 2015). The mesh for these CFD simulations consists of approximately 30,000 to 50,000 hexahedral cells. The transient MPPICFoam solver was chosen to calculate the two-phase flow (Euler-Lagrange). The cyclone simulation from the OpenFOAM cyclone tutorial was used as a basis (OpenFOAM Foundation 2016). The settings for fvSchemes, fvSolution, transportProperties, and turbulenceProperties were adapted to obtain the same setup as for the 3D printing experiments. The settings in the kinematicCloudProperties file were adjusted to the characteristics of the used particles. The density of the particles was changed to $2,700 \mathrm{~kg} / \mathrm{m}^{3}$ (as in Table 1 above). Using the generalDistribution model, the particle distribution from Table 2 can be precisely mapped. In the simulations, 20,000 parcels represent the entirety of the particles, where each parcel has the same mass. The amount of 20,000 parcels was chosen to minimize the represented mass per parcel and not to blow up the calculation time for each timestep. The minimization causes a lower error when a parcel escapes at an outlet.

The heatTransfer, surfaceFilm, damping, stochasticCollision, and radiation submodels were left unchanged at the "off" state. In the experiments, a total of $6 \mathrm{~g}$ was spread over 10 thrusts and the waiting time between each thrust was approximately 3 seconds. The simulation takes only one thrust of $0.6 \mathrm{~g}$ instead of performing the 10 repetitions in order to avoid very long simulation times. The particle velocity in the simulation was set to the same value as the determined velocity of the air at the inlet. Overall, a time frame of 3 seconds is simulated. For this, a total calculation time of approximately 96 hours (wall clock time) using 16 processor cores is required. The simulation was controlled by the time step and relaxation factors and behaved relatively stable.

After each experiment, a certain amount of dust remains in the cyclone. We consider dust as separated, if it leaves the cyclone through the dust exit (usually at the bottom of the cyclone). The evaluation of the simulation results is shown in column (M-C) in Table 5. If the remaining dust in the cyclone is also considered as separated, the collection efficiency is increased.

\subsection{Surrogate Modeling (M-S)}

Computational fluid dynamics simulations are computationally expensive. Data-driven models of cyclone separators, which are substantially cheaper to evaluate, can be used instead. Well-known approaches to handle costly objective functions is to employ response surface or surrogate models (Jin 2003; Kleijnen 2008). That is, data-driven surrogate models may be constructed based on experimental results. Then, an optimization algorithm may work on the surrogate model instead of the actual objective function. To exemplify this approach, 
Table 3 Results from the analytical model (M-A) for cyclone geometries from Hoffmann and Stein (2007). Proportions of the geometries shown in the first three rows (Löffler, Muschelknautz, and Stairmand) were used for the 3D printing experiments. All values were scaled by the height $h_{0}$ of the real cyclone. Since the printed cyclones have an absolute height of $160 \mathrm{~mm}$, the relative values from columns three to nine are multiplied with 160. The Barth model, i.e., Eq. (3), was used to determine the collection efficiency $E$ shown in the last column. It uses the process parameters from our study and the scaled geometry parameters of the original cyclones. For example, the same particle size distribution (silica sand) was used for the calculations to obtain comparable results. The last row shows the geometry parameters and collection efficiency, which were obtained during the metamodel optimization step (S-9) (see Section 7).

\begin{tabular}{lrrrrrrrrl}
\hline Type & $h_{0}[\mathrm{~mm}]$ & $h_{e} / h_{0}$ & $b_{e} / h_{0}$ & $D_{t} / h_{0}$ & $h_{t} / h_{0}$ & $h_{z} / h_{0}$ & $D_{a} / h_{0}$ & $D_{u} / h_{0}$ & $E$ \\
\hline \hline Löffler & 25,000 & 0.2400 & 0.0800 & 0.1680 & 0.2600 & 0.2800 & 0.5040 & 0.1680 & 89.3334 \\
\hline Muschelknautz E. & 9,340 & 0.1852 & 0.0621 & 0.1820 & 0.3330 & 0.1852 & 0.7281 & 0.2441 & 89.9122 \\
\hline Stairmand high eff. & 12,650 & 0.1249 & 0.0498 & 0.1249 & 0.1249 & 0.3747 & 0.2498 & 0.0941 & 89.0490 \\
\hline \hline Muschelknautz D. & 8,630 & 0.2167 & 0.0626 & 0.1379 & 0.3685 & 0.3036 & 0.4137 & 0.2260 & 89.5968 \\
\hline Storch 4 & 16,160 & 0.1609 & 0.0235 & 0.0724 & 0.1089 & 0.5625 & 0.1609 & 0.0563 & 91.4006 \\
\hline Storch 3 & 8,210 & 0.2034 & 0.0731 & 0.1303 & 0.2436 & 0.5627 & 0.2339 & 0.1121 & 86.9871 \\
\hline Storch 2 & 10,970 & 0.1714 & 0.0483 & 0.0985 & 0.2179 & 0.4230 & 0.2051 & 0.0766 & 89.6379 \\
\hline Storch 1 & 19,430 & 0.0515 & 0.0515 & 0.0633 & 0.0726 & 0.2820 & 0.1879 & 0.0329 & 92.4774 \\
\hline Tengbergen C & 9,300 & 0.1075 & 0.1075 & 0.1204 & 0.1559 & 0.2011 & 0.3624 & 0.1204 & 90.4930 \\
\hline Tengbergen B & 6,040 & 0.2964 & 0.0927 & 0.1854 & 0.3709 & 0.5364 & 0.3477 & 0.1854 & 83.8606 \\
\hline Tengbergen A & 6,470 & 0.2087 & 0.1144 & 0.1731 & 0.2427 & 0.2782 & 0.4281 & 0.3122 & 87.7651 \\
\hline TSN -11 & 9,590 & 0.1919 & 0.0563 & 0.1418 & 0.2523 & 0.2284 & 0.3629 & 0.1606 & 89.3807 \\
\hline TSN -15 & 11,240 & 0.1477 & 0.0534 & 0.1406 & 0.3114 & 0.5240 & 0.2367 & 0.1059 & 86.6353 \\
\hline Stairmand high flow & 7,550 & 0.1868 & 0.0940 & 0.1868 & 0.2185 & 0.3748 & 0.2517 & 0.0940 & 81.2173 \\
\hline Van Tongeren AC & 12,310 & 0.1210 & 0.0544 & 0.0812 & 0.2640 & 0.3542 & 0.2640 & 0.1056 & 92.1290 \\
\hline Vibco & 7,200 & 0.1542 & 0.1250 & 0.1542 & 0.1722 & 0.3167 & 0.3972 & 0.0917 & 88.6851 \\
\hline Lapple GP & 11,310 & 0.1247 & 0.0628 & 0.1247 & 0.1565 & 0.5004 & 0.2502 & 0.0628 & 88.8657 \\
\hline \hline Metamodel & 160 & 0.1266 & 0.0235 & 0.0640 & 0.2929 & 0.1853 & 0.7267 & 0.0330 & 94.8474 \\
\hline \hline
\end{tabular}

data from Table 3 was used to fit a simple surrogate model (standard linear regression with variable selection) that models efficiency $E$ as a function 17 different geometries, which were specified in Table 3 (rows 1-17):

$$
E=95.56-0.58 D_{t}+0.11 D_{a} .
$$

The diameter of the vortex finder, $D_{t}$, and the cyclone body diameter, $D_{a}$, have the greatest effect on the collection efficiency. Although this model already shows good accuracy, e.g., adjusted $R$-squared 0.94 , more sophisticated linear models or Kriging models can be fitted (Turner et al. 2013; Kleijnen 2014). Optimization can be performed on this model, e.g., to improve the collection efficiency for a given pressure drop. Even multiobjective optimization techniques can be applied (Elsayed and Lacor 2012; Zaefferer et al. 2014).

\subsection{D-printing Model (M-P)}

3D-printing experiments used standard laboratory equipment: Erlenmeyer flask, stand, pressure gauge, precision scale, and a vacuum cleaner. To ensure comparability and interchangeability of the results, the same design and process parameters as in the other modeling approaches was used for the 3D-printing models. Table 4 shows the parameters of the printed cyclones and process parameters, $\mathbf{x}_{p}$, are described in Ta- ble 1. The model building step (S-4 in the OMMS approach) consists of the (i) 3D computer model generation and the (ii) printing step. The 3D models, described in the STereoLithography, Standard Tessellation Language (STL) are created using a Python script, which uses the FreeCad Python library. ${ }^{1}$ The model is exported to an .STL file, which can be interpreted by a large number of $3 \mathrm{D}$ printers. The printed cyclone models are shown in Figure 3.

Today, a broad variety of 3D printers as well as different materials are available. The printing technique as well as the material have to meet certain requirements. The cyclone has to be robust, because it is fixed into position for the experiments and it has to withstand the flow of air and dust. Due to the hollow shape of the cyclone a ProJet CJP 660pro printer was chosen, which uses gypsum powder (Visijet PXL) as printing substrate. This way, the entire cyclone can be printed in one step. The gypsum powder has to be hardened after surplus gypsum powder has been removed from the interior. Cyan acrylate ("ColorBon") was used to avoid electrostatic charging. It produces a sufficiently smooth surface and improves the stability. Experiments were performed at room temperature. Significantly higher temperatures may require a different choice of material. Printing a single cyclone model takes about three

\footnotetext{
1 http://www.freecadweb.org
} 


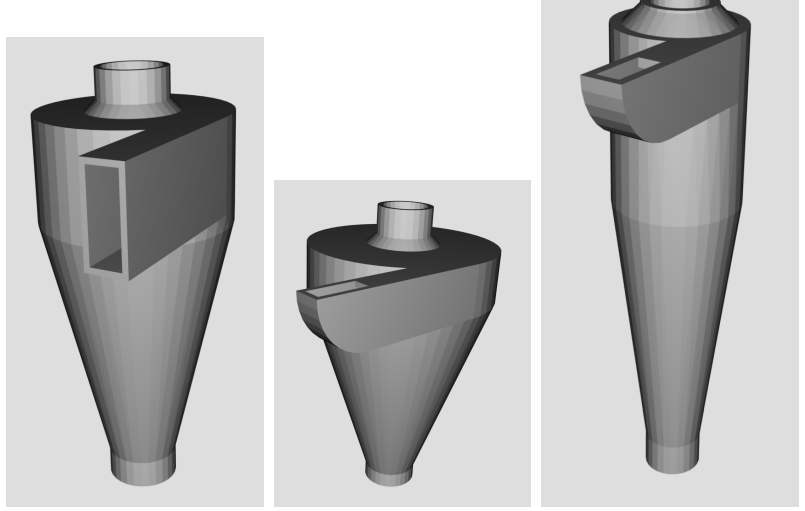

Fig. 3 Three printed cyclone models, which were used with three different $h_{t}$ values in the (M-P) experiments. From left to right: Löffler, Muschelknautz, Stairmand. See Table 4.

hours, plus one hour for refinishing, dust removal, and infiltration, and another hour for curing.

Besides the selection of a printer and material, the characteristics of the dust have to be selected. The distribution of particle sizes should not vary to prevent fluctuations in the results. If the particles are too large, they may be too easy to separate from the gas. If there are too many, they may even block the flow inside the cyclone. If the particles are too small, the task of separation may become near to impossible. The chosen dust is silica sand with a maximal particle size of $63 \mu \mathrm{m}$. Its particle size distribution is shown in Table 2. Three different values for the depth of the outlet pipe $h_{t}$, were chosen for each of the three cyclone shape. Since every experiment was repeated five times, altogether 45 experiments were performed.

\section{Single Models: Experimental Results}

An experimental design was set up to measure the effects of the cyclone shapes and the outlet depth $h_{t}$ on the collection efficiency $E$ in the $3 \mathrm{D}$ printing (M-P) experiments. Three different cyclone geometries, namely Löffler, Muschelknautz, and Stairmand, and three different values $\left(h_{t}=0,35,44 \mathrm{~mm}\right)$ of the outlet pipe immersion were used, see Figure 3. Each experiment was repeated five times. Measurements with unusual collection efficiency values were considered as outliers. For example, an efficiency value larger than $100 \%$ was probably a result of insufficient cleaning of the cyclone between tests. These outliers occurred at the beginning of every series of tests and may be caused by dust particles from previous experiments. Results from these experiments are shown in column (M-P) in Table 5.
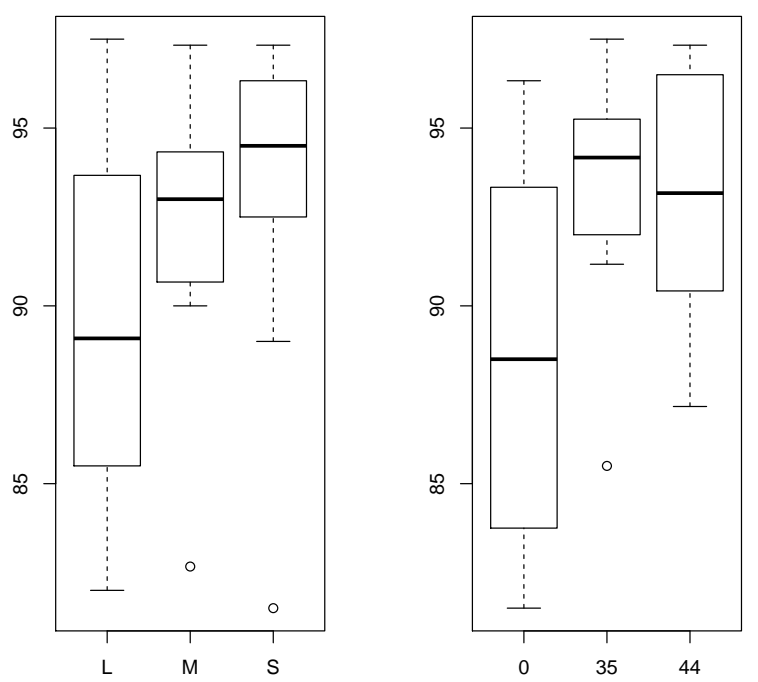

Fig. 4 Collection efficiencies (in \%) of the the three different cyclones (left: Löffler, Muschelknautz, Stairmand) and geometries (right: 0, 35, $44 \mathrm{~mm}$ ) used in the (M-P) experiments. The boxplots indicate that collection efficiency might be improved if Stairmand cyclones and an increased $h_{t}$ value are used. Thick bars indicate the median values.

Figure 4 shows boxplots, which visualize the collection efficiencies of the three different cyclones and outlet pipe immersions. As can be seen in Figure 4, the median of the collection efficiency values of the Löfler cyclone is lower than $90 \%$, whereas it is larger than $90 \%$ for the Muschelknautz and Stairmand cyclones. According to the (M-P) column in Table 5, the Stairmand cyclone has the highest average efficiency. The efficiency at every depth is higher than the previous two cyclones with the same outlet pipe immersions. Furthermore, results from the Stairmand cyclones are more robust, i.e., smaller variances, than results from the other two cyclones. A very small outlet pipe immersion value $(0 \mathrm{~mm})$ results in an reduced collection efficiency as can be seen in Figure 4. This result could be observed consistently for all cyclone types. The results from the 3D printing experiments can be summarized as follows: (i) there are high variances in the measured values, and (ii) the experimental results indicate that the collection efficiency, $E$, increases with increasing vortex finder immersion, $h_{t}$, values.

In addition to the discussion of the results from the $3 \mathrm{D}$ printing experiments, we consider results from the CFD simulations. The experimental CFD results indicate that the collection efficiency is worst if the vortex finder immersion values are zero. This mostly agrees with the findings for the 3D-printed cyclones. In contrast to that observation, data from the analytical model (M-A) show a negative effect of the immersion length, $h_{t}$, on the efficiency: smaller $h_{t}$ values result in 
Table 4 Geometries, i.e., $\mathbf{x}_{g}$ values, of the 3D printed cyclones shown in Figure 3. A total height, $h$ of $160 \mathrm{~mm}$ was chosen. This table shows absolute values, which were determined using the relative values from Table 3 multiplied by 160 .

\begin{tabular}{|c|c|c|c|c|c|c|c|c|}
\hline Type & $b_{e}$ & $D_{a}$ & $D_{t}$ & $D_{u}$ & $h$ & $h_{e}$ & $h_{t}$ & $h_{z}$ \\
\hline Löffler & 12.8 & 80.64 & 26.88 & 26.88 & 160 & 38.4 & 0 & 44.8 \\
\hline Löffler & 12.8 & 80.64 & 26.88 & 26.88 & 160 & 38.4 & 35 & 44.8 \\
\hline Löffler & 12.8 & 80.64 & 26.88 & 26.88 & 160 & 38.4 & 44 & 44.8 \\
\hline Muschelknautz E. & 9.92 & 116.48 & 29.12 & 39.04 & 160 & 29.6 & 0 & 29.64 \\
\hline Muschelknautz E. & 9.92 & 116.48 & 29.12 & 39.04 & 160 & 29.6 & 35 & 29.64 \\
\hline Muschelknautz E. & 9.92 & 116.48 & 29.12 & 39.04 & 160 & 29.6 & 44 & 29.64 \\
\hline Stairmand high eff. & 7.97 & 39.97 & 19.98 & 15.04 & 160 & 19.98 & 0 & 59.95 \\
\hline Stairmand high eff. & 7.97 & 39.97 & 19.98 & 15.04 & 160 & 19.98 & 35 & 59.95 \\
\hline Stairmand high eff. & 7.97 & 39.97 & 19.98 & 15.04 & 160 & 19.98 & 44 & 59.95 \\
\hline
\end{tabular}

Table 5 Summary of the results from different modeling approaches. Collection efficiency $E$ (in \%) as defined in Eq. (3) for three different cyclone types, three different outlet pipe immersions $\left(h_{t}\right)$, and four different modeling approaches (M$\mathrm{A}),(\mathrm{M}-\mathrm{C}),(\mathrm{M}-\mathrm{P})$, and (M-S). 3D-print column (M-P) shows mean (and standard deviation) from five repeats. Obvious outliers were removed. Column (M-S) is based on Eq. (4).

\begin{tabular}{llllll}
\hline Type & $h_{t}$ & $(\mathrm{M}-\mathrm{A})$ & $(\mathrm{M}-\mathrm{C})$ & $(\mathrm{M}-\mathrm{P})$ & $(\mathrm{M}-\mathrm{S})$ \\
\hline \hline Löffler & 0 & 90.19 & 88.57 & $86.80( \pm 4.39)$ & 89.80 \\
Löffler & 35 & 89.49 & 90.91 & $92.13( \pm 5.12)$ & 88.95 \\
Löffler & 44 & 89.27 & 90.19 & $90.84( \pm 3.84)$ & 88.73 \\
\hline Muschelk. & 0 & 91.14 & 82.04 & $88.50( \pm 8.24)$ & 92.13 \\
Muschelk. & 35 & 90.37 & 82.55 & $93.67( \pm 2.17)$ & 91.28 \\
Muschelk. & 44 & 90.15 & 82.43 & $92.87( \pm 2.94)$ & 91.06 \\
\hline Stairmand & 0 & 89.44 & 91.20 & $90.53( \pm 5.68)$ & 88.79 \\
Stairmand & 35 & 88.70 & 95.95 & $94.29( \pm 1.34)$ & 87.93 \\
Stairmand & 44 & 88.45 & 95.60 & $95.50( \pm 2.50)$ & 87.71 \\
\hline \hline
\end{tabular}

an increased collection efficiency $E$. This appears to be a systematic error in the Bart-Muschelknautz method of modeling.

Overall, there are some inconsistencies in the data and in the analytical model that will be considered in forthcoming studies. Some of the required steps to fix these problems are obvious: the variance in the (M-P) model can be reduced by improving the experimental procedure, e.g., by enhancing the cleaning process between the experiments. However, even if the data itself do not enable to draw reliable conclusions for designing an optimal cyclone geometry, they are suitable for demonstrating the OMMS approach.

\section{Metamodel-based Optimization}

A set of surrogate models has to be chosen in step (S7) of the OMMS approach. As level-0 models, a simple regression model $(\mathrm{lm})$, a random forest (rf), and a Kriging $(\mathrm{kr})$ model were used in this study. The metamodel $F^{*}$ from step (S-8) in the OMMS approach can be used for optimizing the geometry parameters $\mathbf{x}_{g}$. This level-1 model, which combines results from the level-0 models, uses the following coefficients:

Ensemble: $-65.02+0.29 \mathrm{~lm}+0.76 \mathrm{rf}-0.77 \mathrm{kr}$,

i.e., the stacked model uses mainly the information from the rf and $\mathrm{kr}$ surrogate (level-0) models, and includes information from the $\mathrm{lm}$ surrogate model to a lesser amount as well. To perform the optimization step, the freely available R package SPOT (sequential parameter optimization toolbox) can be used. It implements several tools for the analysis and optimization of complex problems (Bartz-Beielstein et al. 2005). The SPO function buildEnsembleStack implements the metamodel building step (S-8). After generating an objective function from the fit, an optimizer can be applied (S-9). In our example, differential evolution was used, but any other optimizer is fine.

Results from the optimization are as follows: $h_{e}=$ $0.13, b_{e}=0.02, D_{t}=0.06, h_{t}=0.29, h_{z}=0.19$, $D_{a}=0.73, D_{u}=0.03$. All values are relative to the cyclone height $h_{0}=160 \mathrm{~mm}$. This geometry results in an estimated efficiency of $94.85 \%$. The values are also shown in the last row of Table 3 , allowing a comparison to reported data from the literature. The model suggest a value for the outlet depth $h_{t}$ at medium level. Notably, the efficiency of the proposed solution is lower than some of the single-model results for the cyclone variants shown in Table 5 . These differences are probably caused by noise in the real-world experiments and impreciseness of the models.

The optimization results are compared to the search bounds in Figure 5. This figure illustrates the recommendations from the optimization on the metamodel. For example, the proposed solution requires a minimal diameter of the dust outlet, $D_{u}$, and a minimal diameter of the vortex finder, $D_{t}$. On the other hand, the body diameter, $D_{a}$, should be maximal, and the height of the inlet should have intermediate values.

If the optimization budget is not exhausted, these recommendations can be used to print a new cyclone (M-P) or to perform a CFD simulation and start the next iteration of the OMMS loop. 


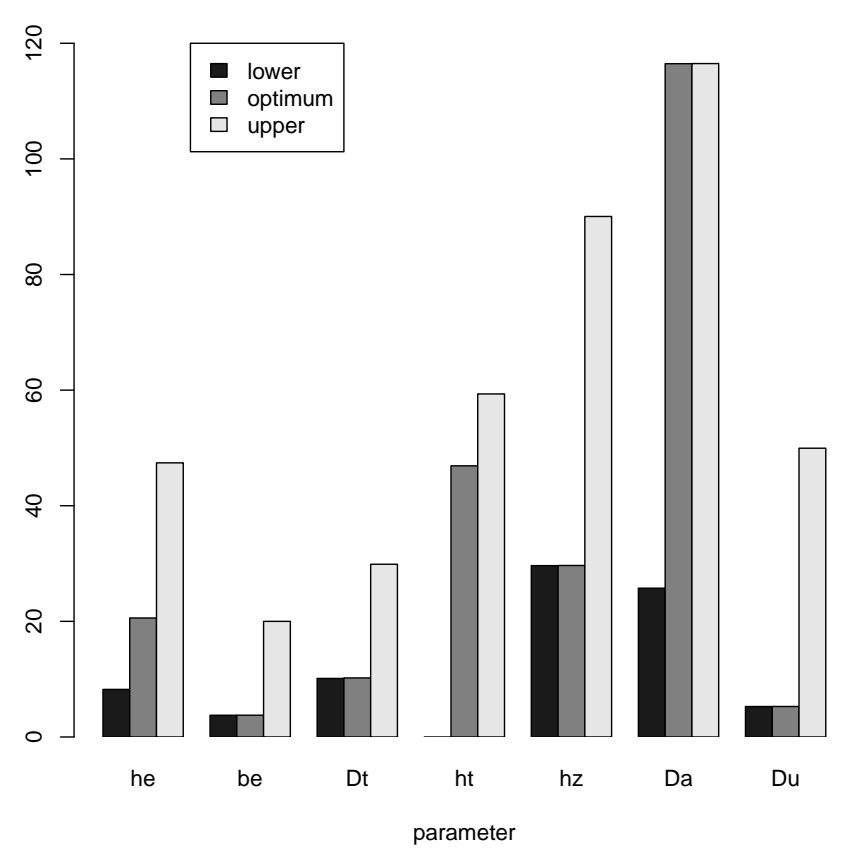

Fig. 5 Optimization on the metamodel (S-9). Barplot of the optimized geometry parameters. Comparing lower and upper bound of decision space with optimum.

\section{Conclusion and Outlook}

This article explores a new approach for combining different simulation approaches. Based on stacking, a flexible methodology for combining results from different models is presented. The experimenters, who carried out the 3D printing experiments, faced complex technical difficulties. Experience from practice plays a crucial role for these experiments. While 3D printing reduces the cost of experiments significantly compared to fullscale simulations, the experiments themselves are still time-consuming and require material resources. With the cost of experiments, noise of evaluation (due to manufacturing as well as measurement inaccuracies), and the inherent complexity of the search-space due to its combinatorics, the modeling based on 3D-printed cyclones poses a major challenge. Due to some technical difficulties and the complexity of the experimental setup, the (M-P) results presented in this study are not yet conclusive. However, we were able to demonstrate how to collect data from (M-P) models and how to combine these results with different modeling approaches. Despite of these problems, we expect that combining results from improved 3D-printing experiments with results from other modeling approaches might improve the overall model quality. This improvement could accelerate the optimization process and lead to new insights into the behavior of cyclones.
This paper can be seen as a proof-of-concept for the OMMS approach, which combines results from several different modeling approaches. It allows the combination of models with different run times. If data is scarce and simulation is expensive, the proposed OMMS approach is a promising way. However, the research question stated in Section 1 cannot be conclusively answered for the experimental setup analyzed in this study. First results, i.e. the interpretation of the improved values from the OMMS as shown in Figure 5, indicate that the weakness of one approach can be compensated by other approaches, but this issue requires further investigation.

How several cheap analytical models can be used for accelerating expensive CFD-based optimization is a highly relevant research question. The Bart-Muschelknautz method of modeling is only one possibility. Currently, more analytical models are added to the SPOT ensemble engine. ${ }^{2}$ The current SPOT package already implements an additional analytical model that is based on Mothes (1984). Due to the technical difficulties of the (M-P) models, alternative model combinations, which include several analytical models, can be recommended. Instead of using the four models from our study, we will combine one CFD model with several analytical und surrogate models.

Last but not least, it should be noted that OMMS cannot be applied without any modifications to every optimization problem. It has to be adapted to the particular application, e.g., cyclone geometry optimization. We do not claim that OMMS outperforms all other algorithms on every problem classes. This is a consequence of the no free lunch theorem (Wolpert and Macready 1997; Haftka 2016).

Acknowledgements This work is part of a project that has received funding from the European Union's Horizon 2020 research and innovation program under grant agreement No. 692286. We would like to thank Horst Stenzel, Beate Breiderhoff, Dimitri Gusew, Aylin Mengi, Baris Kabacali, Jerome Tünte, Lukas Büscher, Sascha Wüstlich, and Thomas Friesen for their support.

\section{References}

Barth, W. (1956). Berechnung und Auslegung von Zyklonabscheidern aufgrund neuerer Untersuchungen. BrennstoffWärme-Kraft, 8(1):1-9.

Bartz-Beielstein, T. (2016). Stacked Generalization of Surrogate Models - A Practical Approach. Technical Report 5/2016, TH Köln, Köln.

2 Source code and data for performing experiments from this study are available at http://www.gm.fh-koeln.de/ $\sim$ bartz/bart16e. The open source R software package SPOT can be downloaded from https://cran.r-project.org. 
Bartz-Beielstein, T., Lasarczyk, C., \& Preuss, M. (2005). Sequential Parameter Optimization. In McKay, B. et al., editors, Proceedings 2005 Congress on Evolutionary Computation (CEC'05), Edinburgh, Scotland, (pp. 773-780), Piscataway NJ. IEEE Press.

Bartz-Beielstein, T. \& Zaefferer, M. (2017). Model-based methods for continuous and discrete global optimization. Applied Soft Computing, 55:154 - 167.

Barzier, M. K. \& Perry, C. J. (1991). An approach to the construction and usage of simulation modeling in the shipbuilding industry. In 1991 Winter Simulation Conference Proceedings., (pp. 455-464).

Chaudhuri, A., Haftka, R. T., Ifju, P., Chang, K., Tyler, C., \& Schmitz, T. (2015). Experimental flapping wing optimization and uncertainty quantification using limited samples. Structural and Multidisciplinary Optimization, 51(4):957-970.

Elsayed, K. \& Lacor, C. (2010). Optimization of the cyclone separator geometry for minimum pressure drop using mathematical models and CFD simulations. Chemical Engineering Science, 65(22):6048-6058.

Elsayed, K. \& Lacor, C. (2012). CFD modeling and multiobjective optimization of cyclone geometry using desirability function, artificial neural networks and genetic algorithms. Applied Mathematical Modelling, 37(8):56805704.

Fishwick, P. A. \& Zeigler, B. P. (1992). A multimodel methodology for qualitative model engineering. $A C M$ Transactions on Modeling and Computer Simulation, 2(1):52-81.

Forrester, A., Sóbester, A., \& Keane, A. (2007). Multi-fidelity optimization via surrogate modelling. Proceedings of the Royal Society A: Mathematical, Physical and Engineering Science, 463(2088):3251-3269.

Fu, M. C. (1994). Optimization via simulation: A review. Annals of Operations Research, 53(1):199-247.

Haftka, R. T. (2016). Requirements for papers focusing on new or improved global optimization algorithms. Structural and Multidisciplinary Optimization, 54(1):1-1.

Haftka, R. T., Villanueva, D., \& Chaudhuri, A. (2016). Parallel surrogate-assisted global optimization with expensive functions - a survey. Structural and Multidisciplinary Optimization, 54(1):3-13.

Hoekstra, A. J., Derksen, J. J., \& Van Den Akker, H. E. A. (1999). An experimental and numerical study of turbulent swirling flow in gas cyclones. Chemical Engineering Science, 54(13-14):2055-2065.

Hoffmann, A. C. \& Stein, L. E. (2007). Gas Cyclones and Swirl Tubes. Springer Berlin Heidelberg, Berlin, Heidelberg.

Jin, R., Chen, W., \& Simpson, T. W. (2001). Comparative studies of metamodelling techniques under multiple modelling criteria. Structural and Multidisciplinary Optimization, 23(1):1-13.

Jin, Y. (2003). A comprehensive survey of fitness approximation in evolutionary computation. Soft Computing, 9(1):3-12.

Kazemi, P., Khalid, M. H., Szlek, J., Mirtič, A., Reynolds, G. K., Jachowicz, R., \& Mendyk, A. (2016). Computational intelligence modeling of granule size distribution for oscillating milling. Powder Technology.

Kleijnen, J. P. C. (2008). Design and analysis of simulation experiments. Springer, New York NY.

Kleijnen, J. P. C. (2014). Simulation-optimization via Kriging and bootstrapping: a survey. Journal of Simulation, 8(4):241-250.
Konan, A. \& Huckaby, D. (2015). Modeling and simulation of a gas-solid cyclone during an upset event (presentation). OpenFOAM Workshop 2015.

Law, A. M. (2007). Simulation Modeling and Analysis. McGraw-Hill, 4 edition.

Löffler, F. (1988). Staubabscheiden. Thieme, Stuttgart.

Meerschaert, M. M. (2013). Mathematical Modeling (Fourth Edition). Elsevier, 4th edition.

Mothes, H. Loeffler, F. (1984). Bewegung und abscheidung der partikel im zyklon. Chem.-Tech.-Ing., 56:714-715.

Murphy, K. P. (2012). Machine learning: a probabilistic perspective. MIT press.

Muschelknautz, E. (1972). Die Berechnung von Zyklonabscheidern für Gase. Chemie Ingenieur Technik, 44(12):63-71.

Nelson, B. L. (1995). Stochastic Modeling: Analysis and Simulation. Dover.

OpenFOAM Foundation (2016). OpenFOAM Tutorials Lagrangian MPPICFoam cyclone. Official OpenFOAM Repository. https://github.com/OpenFOAM/.

Overcamp, T. J. \& Mantha, S. V. (1998). A simple method for estimating cyclone efficiency. Environmental progress, $17(2): 77-79$.

Preen, R. \& Bull, L. (2014). Towards the Coevolution of Novel Vertical-Axis Wind Turbines. Evolutionary Computation, IEEE Transactions on, $\mathrm{PP}(99): 284-294$.

Santner, T. J., Williams, B. J., \& Notz, W. I. (2003). The Design and Analysis of Computer Experiments. Springer, Berlin, Heidelberg, New York.

Simpson, T., Toropov, V., Balabanov, V., \& Viana, F. (2012). Design and Analysis of Computer Experiments in Multidisciplinary Design Optimization: A Review of How Far We Have Come - Or Not. In 12th AIAA/ISSMO Multidisciplinary Analysis and Optimization Conference, (pp. 1-22), Reston, Virigina. American Institute of Aeronautics and Astronautics.

Turner, A. J., Balestrini-Robinson, S., \& Mavris, D. (2013). Heuristics for the regression of stochastic simulations. Journal of Simulation, 7(4):229-239.

Wolpert, D. H. (1992). Stacked generalization. Neural Networks, 5(2):241-259.

Wolpert, D. H. \& Macready, W. G. (1997). No Free Lunch Theorems for Optimization. IEEE Transactions on Evolutionary Computation, 1(1):67-82.

Yang, Y. (2003). Regression with multiple candidate models: selecting or mixing? Statistica Sinica, pages 783-809.

Zaefferer, M., Breiderhoff, B., Naujoks, B., Friese, M., Stork, J., Fischbach, A., Flasch, O., \& Bartz-Beielstein, T. (2014). Tuning multi-objective optimization algorithms for cyclone dust separators. In Proceedings of the 2014 Conference on Genetic and Evolutionary Computation, GECCO '14, (pp. 1223-1230), New York, NY, USA. ACM.

Zeigler, B. P. \& Oren, T. I. (1986). Multifaceted, Multiparadigm Modeling Perspectives: Tools for the 90's. In Proceedings of the 18th Conference on Winter Simulation, (pp. 708-712), New York, NY, USA. ACM. 


\section{Kontakt/Impressum}

Diese Veröffentlichungen erscheinen im Rahmen der Schriftenreihe "CIplus". Alle Veröffentlichungen dieser Reihe können unter

https://cos.bibl.th-koeln.de/home

abgerufen werden.

Die Verantwortung für den Inhalt dieser Veröffentlichung liegt beim Autor.

Datum der Veröffentlichung: 18.03.2018

\section{Herausgeber / Editorship}

Prof. Dr. Thomas Bartz-Beielstein,

Prof. Dr. Wolfgang Konen,

Prof. Dr. Boris Naujoks,

Prof. Dr. Horst Stenzel

Institute of Computer Science,

Faculty of Computer Science and Engineering Science,

TH Köln,

Steinmüllerallee 1,

51643 Gummersbach

url: www.ciplus-research.de

\section{Schriftleitung und Ansprechpartner/ Contact editor's office}

Prof. Dr. Thomas Bartz-Beielstein,

Institute of Computer Science,

Faculty of Computer Science and Engineering Science,

TH Köln,

Steinmüllerallee 1, 51643 Gummersbach

phone: +492261 81966391

url: http://www . spotseven.de

eMail: thomas.bartz-beielstein@th-koeln.de

ISSN (online) 2194-2870

\section{Technology




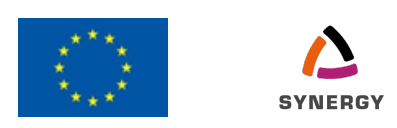

This project has received funding from the European Union's Horizon 2020 research and innovation programme under grant agreement No 692286.

\section{Technology Arts Sciences

